NASA Technical Memorandum 85949

NASA-TM-85949 19840016952

DOR Repmorag

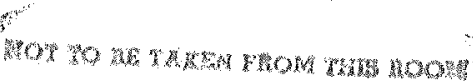

\title{
Optical Measurements of Fluctuating Temperatures in a Supersonic Turbulent Flow Using One- and Two-Photon, Laser-Induced Fluorescence
}

Kenneth P. Gross and Robert L. McKenzie

LERARY COPY

\section{4}

WANGLEY RESEARCH CENTER

LIBRARY, NASA

MAMPTON VIRGINIA 


\section{Optical Measurements of Fluctuating Temperatures in a Supersonic Turbulent Flow Using One- and Two-Photon, Laser-Induced Fluorescence}

Kenneth P. Gross, Polyatomics Research Institute, Mountain View, California Robert L. McKenzie, Ames Research Center, Moffett Field, California

\section{N/SA}


OPTICAL MEASUREMENTS OF FLUCTUATING TEMPERATURES IN A SUPERSONIC TURBULENT FLOW USING ONE- AND TWO-PHOTON, LASER-INDUCED FLUORESCENCE

Kenneth P. Gross*

Polyatomics Research Institute, Mountain V1ew, California

Robert L. Mckenzie ${ }^{\dagger}$

NASA Ames Research Center, Moffett Fleld, California

\section{Abstract}

A laser-induced fluorescence techntque has been developed that provides a practical means of nonintrusively measuring the instantaneous temperatures in low-temperature turbulent flows. The capabilities of the method are reviewed, and its application to a simple, two-dimensional, turbulent boundary-layer flow at Mach 2 is reported. Measurements of the average temperature distribution through the boundary layer and the magnitudes of temperature fluctuations about their average values are presented.

\section{Introduction}

At the present time, few nonintrusive diagnostic methods are available that have sufficient temporal and spatial resolution for measuring the paraneters of interest in fluid dynamics research of cold, turbulent flows. Except for velocity measurements using the well-established laserDoppler velocimetry method, laser-based techniques giving measurements of other. flow fleld properties, such as temperature and density, have not yet been demonstrated that are adequate for the instantaneous sampling of fluctuating quantities. For example, 1ight-scattering processes, such as Rayleigh or spontaneous Raman techniques, are generally restricted to time-averaged measurements; a result of their intrinsically low scattering cross sections and the corresponding difficulties associated with extracting the signal of interest from background noise. 1,2 Modern nonlinear optical methods, such as coherent anti-Stokes Raman scattering (CARS), have been successfully applied primarily to high-temperature combustion environments. However, an alternative optical diagnostic technique that can provide both temporal and spatial resolution in low-temperature fluctuating or turbulent flows is laser-induced fluorescence (LIF), as the results reported here will show.

The sensitive measurement of temperature in low-cemperature flows, using LIF, requires consideration of several fundamental requirements. Those of primary importance include 1) the presence in the flow of a molecular species with absorbing and fluorescing transitions of sufficient strength to provide low-notse signals and signals that are resonant with avallable laser frequencies; 2) the use of spectral features that can be resolved with sufficient detail to accurately infer a temperature; and 3) the availability of sufficient information to accurately account for the effects of fluorescence trapping, collisional quenching, and other fundamental molecular processes affecting the

*Research Scientist.

-Research Scientist. Member AIAA. fluorescence siguals. Generally, to obtain practical spectroscopic measurements of temperature in the range from 150 to $300 \mathrm{~K}$, typical of many gasdynamic test facilities, rotational line spectra must be used because only rotational energy differences are smaller than or comparable to the thermal energy being measured.

Here, we report the application of a laserinduced fluorescence technique, which meets these requirements, to a low-temperature supersonic turbulent flow. To our knowledge, these data are the first of their kind obtained by nonintrusive means with adequate temporal and spatial resolution for turbulent boundary-layer studies. The method, similar to that described previously, ${ }^{3,4}$ requires that the flow be seeded wth a low concentration of nitric oxide. It relies on the detection of the broadband ultraviolet (UV) fluorescence following laser excitation of two transitions orfginating from different ground-state rotational levels in the NO $\left(X^{2} \pi, v^{\prime \prime}=0 \rightarrow A^{2} \Sigma^{+}, v^{\prime}=0\right)$ vibronic $\gamma$ band. Each excitation may be accomplished by using either one-photon or two-photon absorption and each $1 \mathrm{~s}$ induced by a separate tunable laser with its frequency set at the molecular transition of interest. The relattve energy absorbed by each transition is measured by monitoring the subsequent broadband fluorescent emission. The two laser pulses are slightly separated in time, allowing both fluorescence waveforms to be simultaneously recorded with a single detection system. Subsequent deconvolution of the double-pulse waveform allows the individual fluorescence energies to be computed. Since the fluorescence energy is proportional to the energy absorbed by the molecule for each transttion, and since that in turn is proportional to the inftial state number densities, the ratio of fluorescence energies for the two transitions can be related to the rotational temperature of the groundstate NO molecule through the Boltzmann relation for the rotational state number densities:

$$
\frac{I_{2}}{I_{1}} \propto \frac{n_{2}}{n_{1}} a \exp \left[\frac{-\left(E_{2}-E_{1}\right)}{k T}\right]
$$

where $I_{i}$ is the fluorescence intensity, $E_{i}$ is the ground-state rotational energy, $n_{1}$ is the groundstate number density of each transitton, $1, k$ is the Boltzmann constant, and $T$ is the rotational temperature. The rotational temperature thus obtained for the No constituent is closely coupled to the kinetic temperature of the gas mixture, providing repetitive measurements of the local kinetic temperature, acquired at the laser pulse-repetition rate. Simllar fluorescence measurements are also made simultaneously in a nonflowing reference cell at a known pressure and temperature to normalize the varlations in fluorescence slgnal strength owing to pulse-to-pulse fluctuations in laser energy and spectral frequency. 


\section{Experimental Method}

Wind Tunnel

The flow facility used for these experiments is a small blowdown wind tunnel consisting of a high-pressure storage reservoir at ambient. cemperature, a supersonic-nozzle test section, and an evacuated dump tank. The factilty can handle toxic gases such as No at low concentration levels. Up to $300 \mathrm{ppm}$ No in nitrogen was used for the twophoton LIF measurements. The boundary layer studied was produced on the lower wall of a rectangular Mach 2 nozzle. Figure 1 shows a simple schematic of the boundary-1ayer channel and depicts

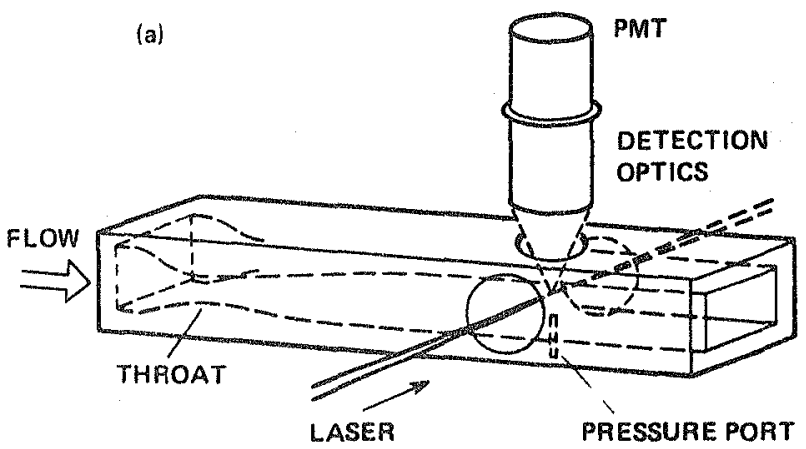

(b)

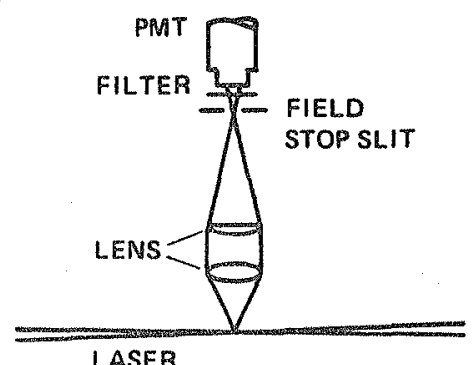

Fig. I Boundary-layer channel. a) Schematic of test section depicting laser access and fluorescence emission ports; b) view of light collection geomerry and imaging system.

the test-section instrumentation ports and collection optics geometry used for the fluorescence measurements. The nozzle has a $25 \times 64-m m$ exit, followed by a slightly diverging channel that extends $762 \mathrm{~mm}$ from the nozzle throat to the measurement ports. The facility was operated at ambient total temperature with stagnation pressures ranging from 3 to $7 \mathrm{~atm}$. At a stagnation pressure of $3.5 \mathrm{~atm}$, the test section contained a fully turbulent boundary layer filling the upper and lower thirds of its 33-m height with an Inviscid core flow in the center. The test time varied from between 5 and $20 \mathrm{sec}$, with the upper limit determined by reservolr stagnation pressure and dump-tank capacity. Optical access to the flow for the lasers was obtained through 50-mm-diam, fusedsilica windows mounted on each side of the channel. Fluorescence was observed through a similar window on the top of the channel. Static pressure measure- ments were made at the channel wall and a pitot tube was temporarily inserted through the top port in the test section to make extensive measurements of total pressure through the boundary layer. Shadowgraphs of the flow taken with and without the pitot tube in place indicated that the measurement volume was free from shock waves or other signiflcant disturbances. The nominal test conditions were as follows.

Stagnation pressure, $P_{t}=3.5 \mathrm{~atm}$

Mach number, $\mathrm{M}_{\infty}=2.03$

Stagnation temperature, $\mathrm{T}_{\mathrm{t}}=295 \mathrm{~K}$

Free-stream temperature, $\mathrm{T}_{\infty}=158 \mathrm{~K}$

Boundary-layer thickness, $\delta=1.4 \mathrm{~cm}$

Reynolds number based on $\delta, \operatorname{Re}_{\delta}=6 \times 10^{5}$

\section{LIF Expertment}

The optical arrangement used for the LIF measurements is illustrated in Fig. 2. Two gratingtuned dye lasers were pumped at $10 \mathrm{kz}$ by the 355-nm third-harmonic output of an Nd:YAG laser. The portion of the pump beam directed to the second dye laser was optically delayed, using two plane mirrors, giving a temporal separation of $130 \mathrm{nsec}$ between the two 5-nsec dye-1aser pulses. Each laser pulse was linearly polartzed, and had an energy of a few millifoules contained in a spectral bandwidth of $0.2-0.3 \mathrm{~cm}^{-1}$.

For two-photon LIF measurements, the first and second dye-laser pulses, at wavelengths near $450 \mathrm{~nm}$, were tuned to half the transitton frequencies corresponding to the $\mathrm{J}^{\prime \prime}=19 \mathrm{I} / 2$ and $\mathrm{J}^{\prime \prime}=7 \mathrm{I} / 2$, S11 + R21, two-photon transitions, respectively. For single-photon measurements, each dye-laser output was frequency-doubled with potasstum pencaborate (KBS) to produce UV energies of several inferojoules. The single-photon transftions used were the $J^{\prime \prime}=181 / 2, Q 11+\mathrm{P} 21$ IIne and the $\mathrm{J}^{\prime \prime}=7 \mathrm{I} / 2, \mathrm{R} 2 \mathrm{I}$ line. In both cases the orthogonally polarized beams were made collinear, focused by a common lens with a 500-mm focal length, and partitioned in the wind-tunnel test section and reference cell. The focal spot size at the measuring point was $0.5 \mathrm{~mm}$.

Broadband nitric oxide fluorescence in the spectral range from 225 to $330 \mathrm{~nm}$ was collected from the flow channel and reference cell with nearly identical f/l fused-silica optics. It was transmitted through broadband UV filters and imaged through an aperture that limited the observed sample volume to a 1-mm path length along the laser beam axis. The fluorescence waveforms were detected by solar-blind photomultipliers, with peak responsivity in the ultraviolet and recorded by Tektronix 7912AD transient digitizers interfaced to an HP- 1000 computer. The effective temporal resolution for each instantaneous temperature measurement was determined by the 130 -nsec separation of dye-laser pulses. Spatial resolution was determined by the nearly cylindrical sample volume of approximately $0.5 \mathrm{~mm}$ dlam by $1.0 \mathrm{~mm}$ in length.

Before each wind-tunnel run, static fluorescence measurements were performed with the flow channel filled with the test gas mixture at ambient 


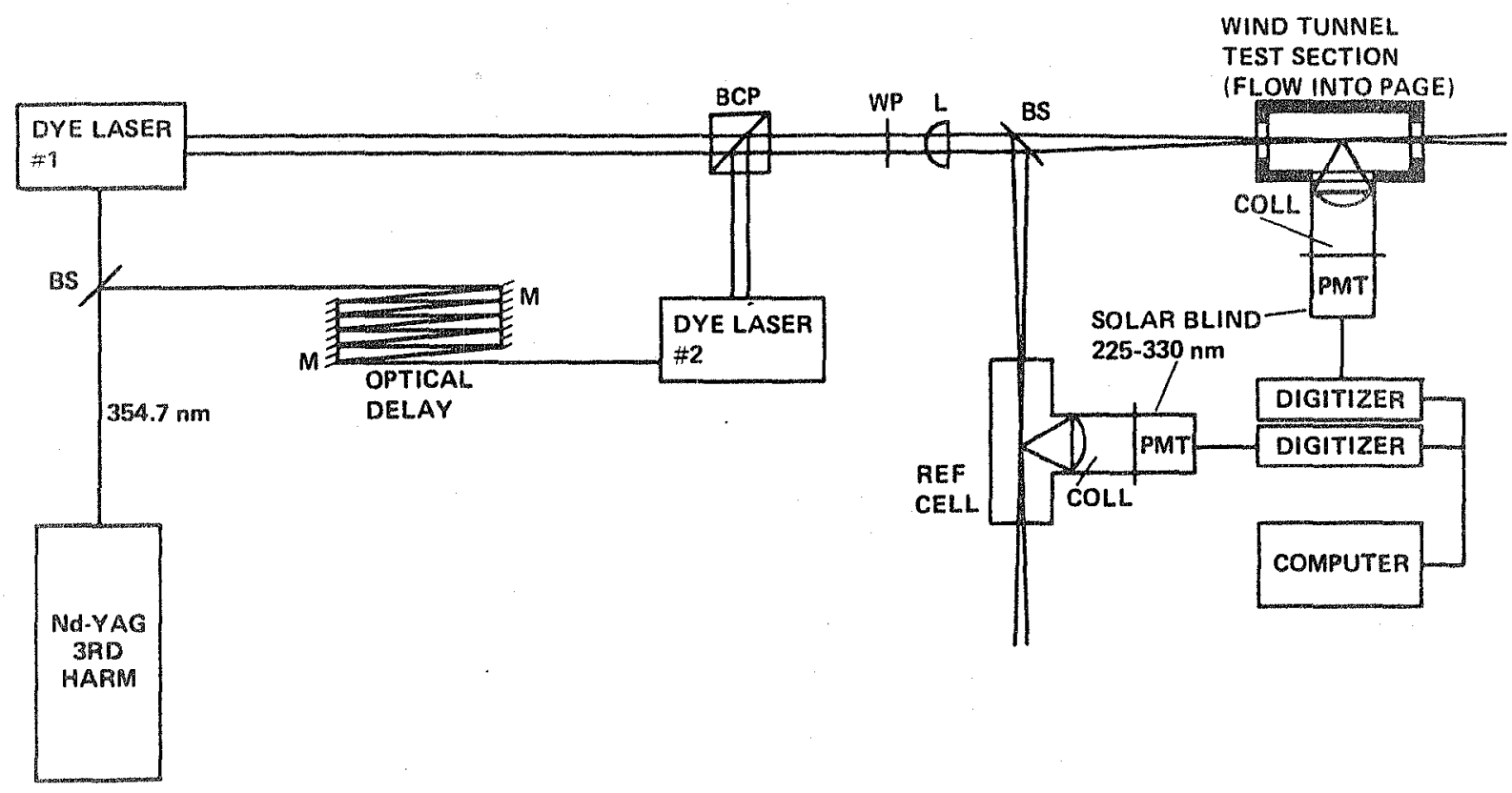

Fig. 2 Schematic of the experimental arrangement: BCP, beam-combining polarizer cube; WP, half-wave plate; $\mathrm{L}$, lens; BS, beamsplitter; $M$, mlrror; COLL, collection system; PMT, photomultiplier tube.

temperature. These pre-run tests served to callbrate the measurement system, using the known pressures and temperatures in both the flow channel and reference cell. Gas pressure in the reference cell was adjusted so that Volgt line-shape proflles were matched to those in the wind-tunnel flow. This procedure maximized the effectiveness for normalization of pulse-to-pulse variations in fluorescence signal amplitude owing to the unavoldable frequency jicter of each dye laser.

As descrtbed in Ref. 3, the data analysis leading to a temperature value for each pump laser pulse requires knowing the ratio of broadband fluorescence energies resulting from each excitation. The ratio is obtained from each double-pulse waverorn by fitting it to a six-parameter function dexived for an exponentially decaying emitter driven by a short excltation pulse with a Gaussian temporal profile. The two pulses in each waveform are then deconvolved and their individual integrals computed. The integral of each pulse is assumed to be lineaxly proportional to the total fluorescence energy resulting solely from its corresponding laser excitation, with account taken of the laser spectral width and all collision-broadened molecular transitions falling within the excitation bandwidth.

Figure 3 shows an example of some experimental fluorescence waveforms and their functional fits For typical wind-tunnel conditions using singlephoton excitation. The first pulse results from excitation of the $J^{\prime \prime}=181 / 2$ rotational level, and the second pulse is from excitation of the $J^{\prime \prime}: 71 / 2$ level. Ultraviolet laser energies were in the range of 1-3 $\mathrm{JJ}$ for each excitation. For an NO concentration of $100 \mathrm{ppm}$ (Fig. 3a), the photonstatistical noise is very low and the signal-tonoise ratios for each pulse were approximately several hundred to one. For the weaker signals at $5 \mathrm{ppm}(\mathrm{F} L \mathrm{~g} \cdot \mathrm{3b})$, the photon-statistical noise on the waveforms is evident and easily distinguished from the computer-fitted function. The experimental waveforms observed using two-photon excitation and 300 ppn No had signal-to-nolse ratios of about 25 , also limited by photon-statistical noise.

\section{Results}

The instrumental uncertainty for the LIF measurements was initially evaluated in a lowtemperature static test cell at conditions dupl1cating those expected in the wind tunnel. The cell contained thermocouples located close to the laser sampling point that provided an independent measurement of the local temperature in the gas mixture. Temperature measurements performed in the static cell using two-photon excitation" are shown in F1g. 4 for a range of temperatures between $155 \mathrm{~K}$ and $295 \mathrm{~K}$. The average temperatures calculated from 50 laser pulses are represented by the circles. The exror bars indicate the corresponding rms deviation from each average value. Generaliy, the average spectroscopic temperatuxes agreed with the thermocouple to within $\pm 2 \%$, whereas the instantaneous single-shot temperatures varied between $3 \%$ and $4 \% \mathrm{rms}$ for each data set, thus defining the instrumental rms noise sensitivity using two-photon excitation.

Two-photon excitation of the NO $\gamma(0,0)$ band, although easier and more convenient to implement experimentally than single-photon excitation, suffers from stark broadening caused by the intense laser fields needed to induce appreciable absorption. ${ }^{4}, 5$ This effect, which manifests itself as a power-dependent broadentng of the spectroscoptc lines used for the measurement, defeats the use of normalization by a reference cell, and forces the measurements to be made using a minimum laser intensity with a corresponding increase in signal notse. It is this basic limitation for the two-photon process that limits the temperature measurement noise to approximately $4 \%$. By using single-photon excitation instead of a multiphoton scheme, much higher signal levels can be achieved with much lower laser 

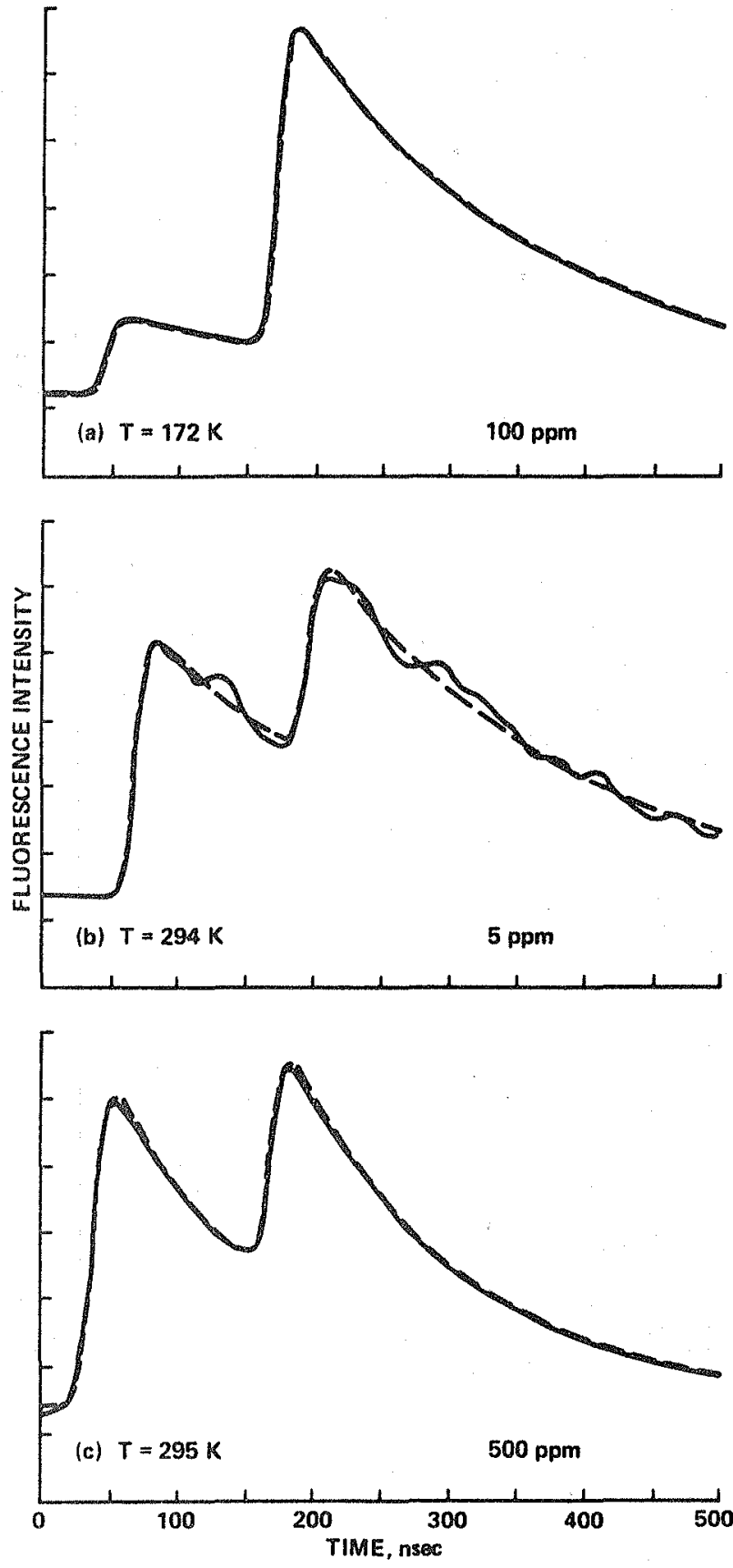

Fig. 3 Dual-pulse fluorescence waveform (solid line) and computer-fitted function (dashed line). a) Boundary-layer sample with $100 \mathrm{ppm}$ NO, static pressure $=0.43 \mathrm{~atm}, \mathrm{~T}=172 \mathrm{~K}$; b) room-temperature sample with $5 \mathrm{ppm}$ No in $0.5-\mathrm{atm} \mathrm{N}_{2}, \mathrm{~T}=294 \mathrm{~K}$; c) room-temperacure reference cell with $500 \mathrm{ppm}$ NO in $0.6 \mathrm{~atm} \mathrm{~N}, T=295 \mathrm{~K}$. First pulse is fluorescence from the one-photon Q11 + P21 (18 1/2) excitation; second pulse is from the R21 (7 1/2) excitation. Vertical amplifier bandwidth, $20 \mathrm{MHz}$; sweep, 50 nsec/div.

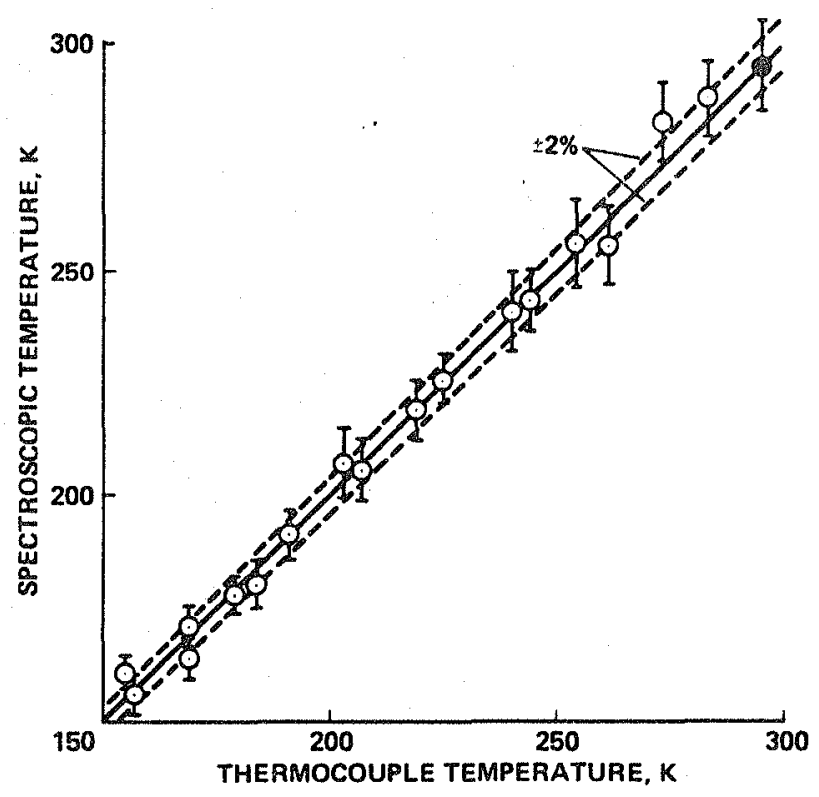

Fig. 4 Comparison of measured rotational temperature and thermocouple temperature using two-photon excitation. Cell mixture: $300 \mathrm{ppm}$ No in 0.5 atm $\mathrm{N}_{2}$ 。

Intensities, giving the added advantage that Stark effects are completely negliglble. The primary disadvantages accompanying the use of one-photon excltation are the need for a more complicated experimental setup, the practical difficulties assoclated with handing and directing ultraviolet laser beams, and the rejection of scattered laser light at wavelengths close to those of the fluorescence signals. Fortunately, for the case of No $Y(0,0)$ band excitation, the single-photon-induced fluorescence stgnals are much larger than the stray light levels that were encountered in these experiments, allowing adequate rejection of radiation at the excitation frequency with simple band-pass filters.

The higher stgnal levels produced using singlephoton excltation also allow a considerable reduction in nitric oxide seeding concentration. F1gure 5 is a plot of temperature sensitivity versus NO concentration, using UV laser energies of a few microjoules. It shows that for nitric oxide concentrations above $50 \mathrm{ppm}$ in $0.5-\mathrm{atm} \mathrm{N}_{2}$, the rms noise in the temperature measurement is about $1 \%$. At concentrations below $50 \mathrm{ppm}$, the effects of photon-statistical noise begin to increase. Nevertheless, useful measurements can still be made at concentrations of only a few ppm, although interference from scattered laser light w111 become a larger fraction of the total signal and may require increased spectral. filtering. Single-photon measurements using concentrations of about $1-2 \mathrm{ppm}$ are nearly equivalent in noise sensitivity to the corresponding two-photon measurements made using 300-ppm NO.

The results of the wind-tunnel tests are 11lustrated in Figs. 6 and 7 . Figure 6 shows the distribution of average temperature obtained by the LIF method, as the measurement point is traversed from the channel centerline to a position approximately $0.5 \mathrm{~mm}$ from the wall. Both one-photon and 


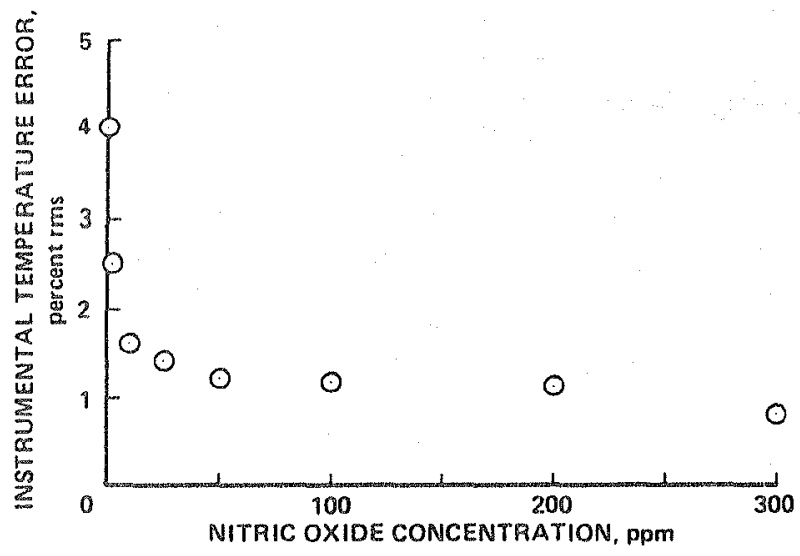

Fig. 5 Instrumental temperature error as a function of nitric oxide concentration, using singlephoton excitation in a nonflowing cell at anbient remperature: $\quad P=0.5 \mathrm{~atm}$.

\section{O IMPLIED FROM IMPACT PRESSURE PROBE 1.PHOTON AND 2.PHOTON LIF}

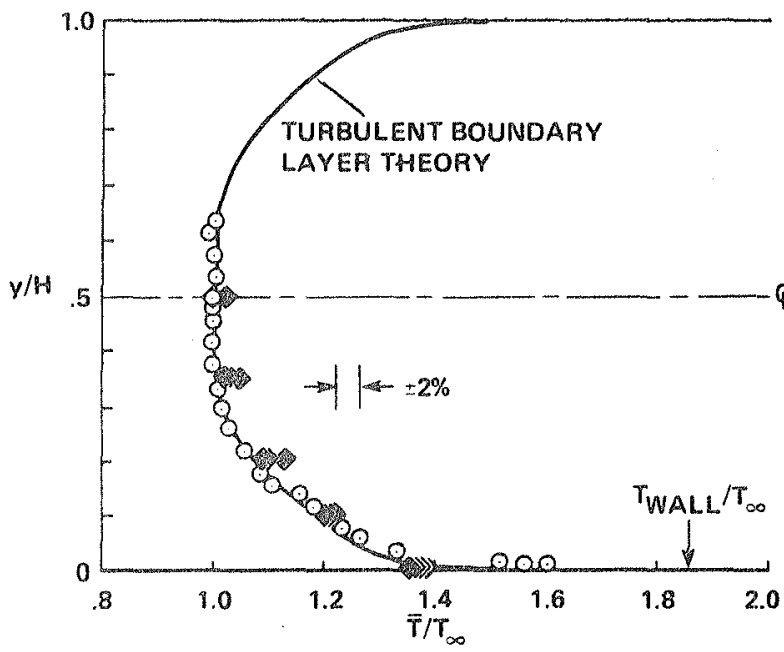

Fig. 6 Average temperature distribution through the boundary layer: channel height $\mathrm{H}=32.5 \mathrm{~mm}$; $\mathrm{M}_{\infty}=2 ; \mathrm{T}_{\mathrm{t}}=295 \mathrm{~K} ; \mathrm{T}_{\infty}=158 \mathrm{~K}$.

two-photon data are presented together and are show by the solid symbols. They represent averages accumulated during $13-\mathrm{sec}$ runs, with account taken of the declining stagnation temperature that is characteristic of blowdown flow facilities. The values plotted are the corresponding average temperatures after 2 sec from the start of the run. The data-acquisition rate was limited by the digitizers to $5 \mathrm{~Hz}$, corresponding to 65 temperature determinations per run. The open symbols are temperatures implied from a pltot probe survey at the same conditions, using the standard onedimensional equations derived for isentroptc comm pressible flow ${ }^{6}$ and assuming that the total temperature and static pressure are constant through the boundary layer. The solid theoretical curve is a solution of the compressible, turbulent, boundary-1ayer equations using a two-layer algebraic eddy-viscosity model. ${ }^{7}$ Average temperatures

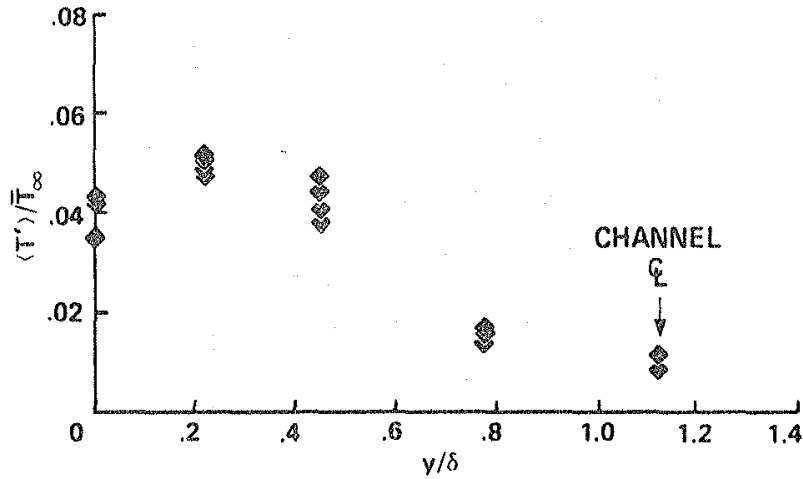

Fig. 7 Distribution of rms temperature fluctuations tin the boundary layer using single-photon LIF; $y / \delta$ is the location perpendicular to the wall normalized to the boundary-layer thickness. Gas mixture, $100 \mathrm{ppm}$ NO; $\mathrm{T}_{\infty}=158($ Mach 2); $\delta=1.4 \mathrm{~cm}$.

determtned using either one- or two-photon excitation are in agreement with one another and both agree with the pitot probe results to with1n $\pm 2 \%$.

Figure 7 depicts the rms magnitudes of the temperature fluctuations owing to turbulence, obtained by single-photon LIF for an No concentration of $100 \mathrm{ppm}$. The total rms deviations have been corrected by removing the average instrumental noise of $\approx 1 \%$ determined from the static calibration runs, thus leaving only the fluctuating temperature component resulting from the local flow conditions. Run-to-run vartations of the turbulence level determined at the same location in the boundary layer may be due in part to statistical uncertainties resulting from the linited number of data samples raken during a given wind-tunnel run.

\section{Conclusion}

Quantitative nonintrusive measurements of fluctuating temperatures using laser-induced fluorescence have been made in a simple, wellcharacterized, Mach 2, curbulent boundary-layer flow, seeded with 100-300 ppr of NO. Results obtained using both one-photon and two-photon No $y(0,0)$ rotational line excttation agree with average temperature profiles deduced from pitot-probe surveys to within $2 \%$. The method ustng two-photon LIF has been shown to be wuch less sensitive for instantaneous measurements than the one-photon method, a result of optical stark broadening caused by the high laser fleld intensiries needed for the twom photon measurements. The application of one-photon LIF in this study has demonstrated the capability for directly measuring turbulent fluctuations of temperature as small as $1 \%$ of the average local value. Additionally, the one-photon technique remains fairly sensitive at much lower No concentrations, and could be effectively implemented in larger scale facilities where lower seeding levels are more desirable.

Work in progress in our laboratory indicates that the present method can most likely be extended to include simultaneous density measurements using an off-resonance excitation approach similar to that described for flow studies using todine fluorescence. ${ }^{B}$ This refinement would allow 
simultaneous measurements of temperature and density, thus providing nonintrusive pressure measurements.

\section{Acknowledgments}

The authors are grateful to D. J. Monson and P. Logan for providing the pitot tube measurements and boundary-layer calculations represented in Fig. 6 .

\section{References}

${ }^{1}$ Peterson, C. W., "A Survey of the Utilitartan Aspects of Advanced Flowfield Diagnostic Techniques," AIAA Journal, Vol. 17, Dec. 1979, pp. $1352-1360$

${ }^{2}$ Lederman, S. and Sacks, S., "Laser Diagnostics for Flowfields, Combustion, and MHD Applications," AIAA Journa1, Vo1. 22, Feb. 1984, pp. $161-173$.

${ }^{3}$ McKenzie, R. L. and Gross, K. P., "TwoPhoton Excitation of Nitric Oxide Fluorescence as a Temperature Indicator in Unsteady Gasdynamic Processes," Applied Opt1cs, Vol. 20, 1981, p. 2153.
"Gross, K. P. and McKenzie, R. L., "SinglePulse Gas Thermometry at Low Temperatures Using Two-Photon Laser-Induced Fluorescence in NO-N Mixtures," Optics Letters, Vol. 8, 1983, p. 368.

${ }^{5}$ Huo, W. M., Gross, K. P., and McKenzie, R. L., "Opt1cal Stark Effect in the Two-Photon Spectrum of No," to be submitted to Phystcal Review Letters.

6 "Equations, Tables, and Charts for Compressible Flow," NACA-1135, 1953.

7 "Boundary Layer Integral Matrix Procedure, Version C (BLIMPC)," Aerotherm Report No. UM-70-20, June 22, 1970.

${ }^{8}$ McDanie1, J. C., Baganoff, D., and Byer, R. L., "Density Measurement in Compressible Flows Using off-Resonant Laser-Induced Fluorescence," Physics of Fluids, Vo1. 25, 1982, p. 1105. 


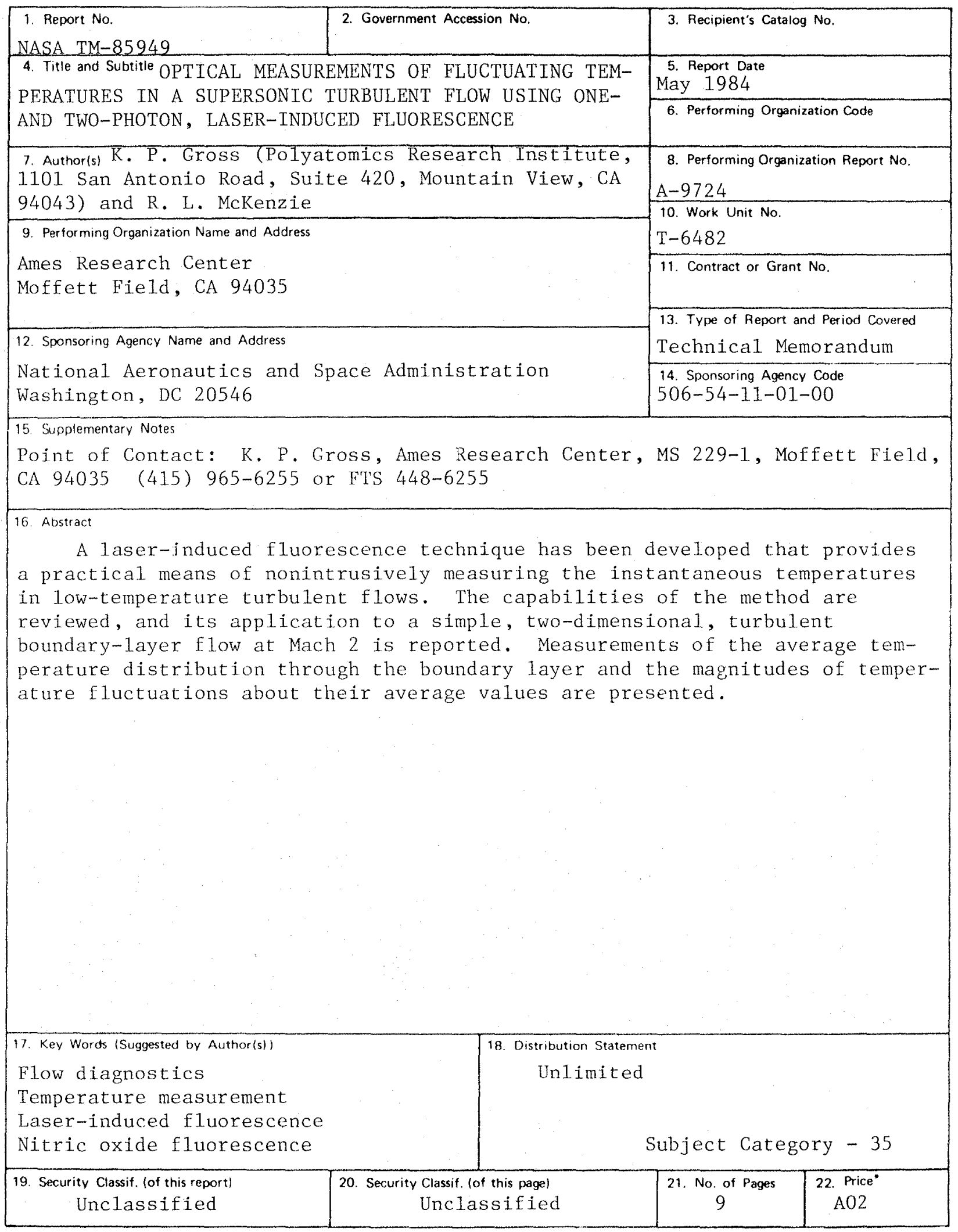

"For sale by the National Technical Information Service, Springfield, Virginia 22161 
End of Document 